\title{
Induction of Neonatal Tolerance by Plasmid DNA Vaccination of Mice
}

\author{
Gil Mor, ${ }^{\star}$ Galina Yamshchikov, ${ }^{*}$ Martha Sedegah, ${ }^{\S}$ Mitsuhiro Takeno, ${ }^{\star}$ Ruobing Wang, ${ }^{\S}$ Richard A. Houghten, ${ }^{\ddagger}$ \\ Stephen Hoffman, $\$$ and Dennis M. Klinman* \\ * Section of Retroviral Immunology, Center for Biologics Evaluation and Research, Food and Drug Administration, Bethesda, Maryland \\ 20892; ${ }^{\ddagger}$ Torrey Pines Institute of Molecular Studies, San Diego, California 92121; and ${ }^{\S}$ Malaria Program, Naval Medical Research \\ Institute, Bethesda, Maryland 20889
}

\begin{abstract}
Plasmid DNA vaccines capable of preventing viral, bacterial, and parasitic infections are currently under development. Our labs have shown that a plasmid DNA vaccine encoding the circumsporozoite protein of the malaria parasite elicits protective immunity against live sporozoite challenge in adult $\mathrm{BALB} / \mathrm{c}$ mice. We now find that the same DNA vaccine induces tolerance rather than immunity when administered to 2-5 d-old mice. Neonatally tolerized animals were unable to mount antibody, cytokine or cytotoxic responses when rechallenged with DNA vaccine in vitro or in vivo. Tolerance was specific for immunogenic epitopes expressed by the vaccine-encoded, endogenously produced antigen. Mice challenged with exogenous circumsporozoite protein produced antibodies against a different set of epitopes, and were not tolerized. These findings demonstrate important differences in the nature and specificity of the immune response elicited by DNA vaccines versus conventional protein immunogens. (J. Clin. Invest. 1996. 98: 2700-2705.) Key words: DNA vaccine • tolerance • malaria • neonate • epitope
\end{abstract}

\section{Introduction}

Infectious organisms are a major source of morbidity and mortality worldwide. Preventing these infections is a public health priority and the primary goal of vaccine research. New vaccine development has been revolutionized by the finding that antigen-encoding DNA plasmids can induce cellular and humoral immune responses against pathogenic viruses, parasites and bacteria (1-5). DNA vaccines successfully prevent infection in a variety of animal models, and are currently undergoing phase I clinical trials in humans. These vaccines are composed of an antigen-encoding gene whose expression is regulated by a strong mammalian promoter expressed on a plasmid backbone of bacterial DNA $(1,6)$. When injected intramuscularly (i.m.) or intradermally (i.d.), DNA vaccines are transcribed, translated, and the protein they encode presented to the immune system in the context of self $\operatorname{MHC}(1,6,7)$.

A variety of DNA vaccines (administered i.m. or i.d.) induce strong protective immune responses in adult animals (1$3,8,9)$. Yet most vaccines intended for human use are admin-

Address correspondence to Dennis Klinman, Bldg. 29A Rm. 3 D10 HFM 454, CBER/FDA, Bethesda, MD 20892. Phone: 301-827-1707; E-mail: klinman@A1.cber.fda.gov

Received for publication 31 July 1996 and accepted in revised form 26 September 1996.

The Journal of Clinical Investigation

Volume 98, Number 12, December 1996, 2700-2705 istered to infants and children. Due to the immaturity of their immune system, newborns exposed to foreign antigens are at risk of developing tolerance rather than immunity (10). A number of factors influence the development of neonatal tolerance, including the nature, concentration and mode of antigen presentation to the immune system, and the age of the host (11-13). Evidence suggests that recognition of foreign determinants is acquired at distinct stages of maturation, ranging from early gestation until days or weeks after birth $(11,12,14$ 16). Since the protein encoded by a DNA vaccine is produced endogenously and expressed in the context of self MHC, the potential exists for the neonatal immune system to recognize it as 'self,' resulting in tolerance rather than immunity.

Our research focused on a DNA vaccine encoding the circumsporozoite protein of the Plasmodium yoelii (PyCSP $)^{1}$ malaria parasite. This pCSP DNA vaccine induces an antibody and CTL response that protects adult $\mathrm{BALB} / \mathrm{c}$ mice from subsequent challenge with live sporozoites (3), and thus is considered an important model for human antimalarial vaccine development. Unlike its effect in adults, we find the pCSP DNA vaccine induces long-lasting tolerance when administered to newborn mice.

\section{Methods}

Reagents. The pCSP DNA vaccine was constructed by cloning the DraI-EcoRV fragment of the PyCSP gene into the HincII site of pBluescript II SK $(+)$ (Stratagene Inc., La Jolla, CA), and then transferring it into the Sal I/Klenow-filled and BamHI sites of a kCMVinBL vector (a modified pUC18-based plasmid pCMVintBl [7]) where the ampicillin-resistance gene was replaced with a kanamycin resistance gene by using the pBluescript XhoI/Klenow-filled and BamHI restriction endonuclease sites located $5^{\prime}$ and $3^{\prime}$, respectively, to the PyCSP coding sequence, as previously described (3). Expression of PyCSP was tested by in vitro transfection of BHK cells and immunoblot analysis of cell lysates. Plasmid DNA was purified by cesium chloride gradient centrifugation, sterilized by ethanol precipitation, and demonstrated to be endotoxin-free before being dissolved in sterile PBS for injection.

A series of $P$. yoelii CS protein derived antigens were used in ELISA, CTL, and/or in vivo stimulation experiments. CS.1 is an immunoaffinity-purified fusion protein produced in Escherichia coli, and consists of amino acids 64-321 of the intact PyCSP protein fused to $81 \mathrm{AA}$ of the nonstructural protein of influenza A $(3,17)$. A series of 17 synthetic peptides were used to map the epitope specificity of serum antibodies reactive with PyCSP. The AA sequence of peptides recognized by anti-CS antibodies was: peptide 3, GYGQNKSNQAQRNLNELCYN; peptide 9, DDPPKDGNKDDLPKEEKKDD;

1. Abbreviations used in this paper: CS.1, recombinant-purified circumsporozoite protein; pCSP, plasmid DNA vaccine encoding the PyCSP protein; PyCSP, circumsporozoite protein of Plasmodium yoelii. 
peptide 10, DLPKEEKKDDLPKEEKKDDP; peptide 14, VVADENVDQGPGAPQGPGAP; peptide 15, QGPGAPQGPGAPQGPGAP; peptide 19, YVPSAEQILEFVKQISSQLT; peptide 21, EEWSQCSVYCGSGVRVRKRK; peptide 22, GSGVRVRKRKNVNKQPENLT; peptide 25, MDKCSSIFNIVSNSLGFVIL, and the CTL epitope P16, SYVPSAEQILEFVKQI (AAs 281-296).

Animals. Female BALB/c mice were bred and maintained in the specific pathogen animal colony at the Center for Biologics Evaluation and Research. Mice were immunized between $2 \mathrm{~d}$ and $6 \mathrm{wk}$ of age and boosted from $6 \mathrm{wk}$ to 6 mo of age. Adults were immunized and boosted in both quadriceps muscles with $40 \mu \mathrm{g}$ of plasmid DNA, while $10 \mu \mathrm{g}$ of vaccine was injected into the gluteus maximus of mice under $1 \mathrm{wk}$ of age. $50 \mu \mathrm{g}$ of CS.1 protein emulsified in Complete Freund's adjuvant was injected intraperitoneally (i.p.) into adult mice, while $20 \mu \mathrm{g}$ of soluble CS.1 was injected i.p. into newborns (18).

3-6 wk after immunization, animals were bled by retro-orbital puncture, killed by cervical dislocation, and their spleens removed aseptically. Sera was stored at $-70^{\circ} \mathrm{C}$ and assayed for antibody by ELISA while freshly isolated cells were examined for cytokine production.

Cytokine ELIspot assays. 96-well Immulon 2 microtiter plates were coated with $10 \mu \mathrm{g} / \mathrm{ml}$ of anti-IFN $\gamma$ (clone RA6a2, Lee Bimolecular), or anti-IL-4 (clone DVD4-1D11, Endogen, Boston, MA) in 0.1 M carbonate buffer ( $\mathrm{pH}$ 9.6) for $3 \mathrm{~h}$ at room temperature (19). The plates were blocked with PBS-5\% BSA for $1 \mathrm{~h}$ and washed with PBS- $0.025 \%$ Tween 20.

Spleen cell suspensions were prepared in complete medium (RPMI-1640 supplemented with 10\% heat inactivated FCS, $1.5 \mathrm{mM}$ L-glutamine and $100 \mathrm{U} / \mathrm{ml}$ of penicillin/streptomycin) as described (20). Serial dilutions of single cells suspensions, starting with $1-10 \times 10^{5}$ cells/well, were incubated on anticytokine-coated plates in complete medium for $8-10 \mathrm{~h}$ at $37^{\circ} \mathrm{C}$ in a humidified $5 \% \mathrm{CO}_{2}$ incubator. In some cases, PyCSP-derived T cell stimulatory peptides were included during the assay period. Plates were then washed with PBS-Tween and overlaid with $1 \mu \mathrm{g} / \mathrm{ml}$ of biotinylated anti-IFN $\gamma$ (clone XMG 1.2, Pharmingen, San Diego, CA) or anti-IL-4 (clone BVD6-24G2, Endogen) at $4^{\circ} \mathrm{C}$ and then treated with a 1:2,000 dilution of avidin-conjugated alkaline phosphatase (Vector Laboratories, Burlingame, CA) for $2 \mathrm{~h}$ at RT. After a final wash, individual cytokine-secreting cells were visualized by the addition of a solution of BCIP/NBT (Kirkegaard and Perry Labs, Gaithersburg, MD).

Peptide and CS.1-specific ELISA assays. 96-well Immulon $1 \mathrm{mi}-$ crotiter plates were coated with $10 \mu \mathrm{g} / \mathrm{ml}$ of immunoaffinity purified CS.1 protein or CS.1-derived synthetic peptides (sequences shown above) in carbonate buffer, $\mathrm{pH} 9.5$ (21). Plates were blocked with PBS $1 \%$ BSA, overlaid with serially diluted mouse serum, washed, and reacted with phosphatase-conjugated anti-mouse IgG (Southern Biotechnologies, Birmingham, AL). The concentration of specific antibody was determined by comparison to a standard curve generated using a high-titered anti-serum.

Cytotoxic T cell studies. CTL assays were performed as described (22). Briefly, spleen cells from control or immunized mice were cultured at a density of $2 \times 10^{6} / \mathrm{ml}$ for $5 \mathrm{~d}$ with $2.5 \mu \mathrm{M}$ of peptide encoding the PyCSP CTL epitope. These cultures were maintained in complete medium that was supplemented with IL-2 on day 2. P815 mastocytoma $\left(\mathrm{H}-2^{\mathrm{d}}\right)$ and EL-4 thymoma $\left(\mathrm{H}-2^{\mathrm{b}}\right)$ targets were incubated with $2.5 \mu \mathrm{M}$ of the CTL peptide, a control peptide from the Plasmodium falciparum CSP, or no peptide for $18 \mathrm{~h}$ before assay (3, 23). These targets were labeled with $0.1 \mathrm{mCi}$ of ${ }^{51} \mathrm{Cr}$ (New England Nuclear, Boston, MA) and incubated at $37^{\circ} \mathrm{C}$ for $6 \mathrm{~h}$ on the day of assay. 5,000 labeled targets were washed three times and incubated at various ratios with effector cells in 96-well U-bottom plates. Supernatants were harvested after $6 \mathrm{~h}$ and ${ }^{51} \mathrm{Cr}$ release measured. Net specific lysis was calculated by the formula: (percent lysis of cells pulsed with the PyCSP-specific peptide - percent lysis of cells pulsed with the unrelated peptide). All assays were carried out in triplicate.

Data analysis. All results represent the average of $\geq 4$ individually tested mice/group. Statistical significance was established using Student's $t$ test.

\section{Results}

Humoral immune response to the pCSP DNA vaccine. The pCSP DNA vaccine incorporates the gene encoding the circumsporozoite protein (CSP) of the $P$. yoelii malaria parasite into the 1012 plasmid vector. When administered i.m. to adult $\mathrm{BALB} / \mathrm{c}$ mice, the pCSP vaccine induces a strong IgG antiPyCSP antibody response (Fig. 1, lines 1 and 2) whereas no response was elicited by the 1012 plasmid vector (Fig. 1, line 3). Yet when administered to $2-5 \mathrm{~d}$-old BALB/c mice, this vaccine failed to elicit anti-PyCSP antibodies (Fig. 1, line 4). Similarly, no antibody response was detected when neonatal mice were immunized with the recombinant circumsporozoite fusion protein, CS.1 (Fig. 1, line 5). These findings suggest that newborns either do not recognize, or are tolerized by, the circumsporozoite protein.

To discriminate between these alternatives, mice were injected with pCSP or CS.1 at $2 \mathrm{~d}$ of age and then reimmunized as adults. Mice treated as neonates with CS.1 protein generated strong anti-PyCSP antibody responses when immunized/ boosted with CS.1 emulsified in Complete Freund's Adjuvant (CS.1/CFA) or the pCSP DNA vaccine as adults (Fig. 1, lines 6 and 7). This demonstrates that neonatal mice are not tolerized

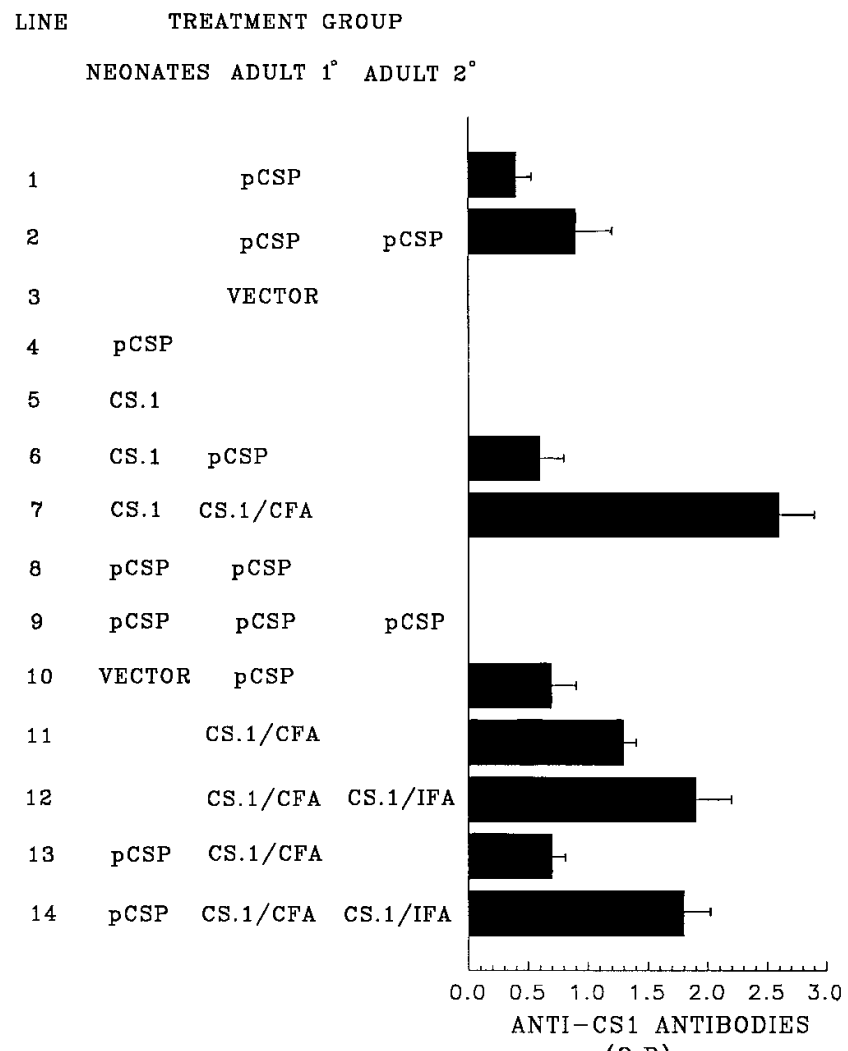

(O.D)

Figure 1. BALB/c mice were immunized in both quadriceps muscles with pCSP, the plasmid vector from which it was derived, or CS.1 protein. $3 \mathrm{wk}$ after immunization, a 1:100 dilution of serum from these animals was assayed by ELISA for IgG antibodies reactive with CS.1. Data represent the mean optical density \pm SD of sera from three to nine individually tested animals/group. The absence of PyCSP-specific antibodies in neonatally tolerized mice was confirmed by indirect fluorescent antibody testing of whole fixed malaria sporozoites ([3], data not shown). 
by exogenous CS.1 protein. In contrast, neonatal mice treated with pCSP were unresponsive to subsequent immunization or boosting (at 6 wk to 9 mo of age) with the pCSP DNA vaccine (Fig. 1, lines 8 and 9). This lack of reactivity was uniquely associated with the administration of pCSP to neonatal mice, since (a) neonatal mice treated with 1012 vector (lacking the CSPencoding gene) were not tolerized to pCSP (Fig. 1, line 10) and (b) the same vaccine administered to adult mice was highly immunogenic (Fig. 1, lines 1 and 2). We thus conclude that the pCSP DNA vaccine (unlike soluble CS.1 protein) induces tolerance rather than immunity when administered to neonatal mice.

Epitope specificity of anti-CSP antibodies. To identify the epitopes that elicited humoral tolerance in pCSP-treated neonatal mice, adult animals were immunized with pCSP or CS.1/ CFA. Sera obtained 3 and 6 wk after immunization were screened for reactivity against a panel of 17 synthetic peptides spanning the length of the PyCSP protein (23). Peptide 25 was recognized by sera from both $\mathrm{pCSP}$ and CS.1/CFA immunized mice (Fig. 2, lines 1-4). By comparison, peptides 3, 19, 21, and 22 were uniquely recognized by sera from mice immunized with the pCSP DNA vaccine while peptides 9, 10, 14, and 15 were uniquely recognized by sera from CS.1/CFA-immunized animals (Fig. 2, lines 1-4). The remaining peptides were not recognized by any sera. These observations indicate that immune recognition of epitopes on the circumsporozoite protein differed among mice immunized with the pCSP DNA vaccine versus exogenous CS.1 protein.

Although no anti-PyCSP response was elicited when mice tolerized as neonates with pCSP were immunized or boosted with that DNA vaccine as adults (Fig. 2, line 5), neonatally tolerized mice were able to produce IgG anti-PyCSP antibodies when immunized with CS.1 protein emulsified in CFA (Fig. 1, lines 9 and 10). Of interest, antibodies from such mice bound to peptides 9, 14, and 15, but not peptide 25 (Fig. 2, line 6). These findings indicate that tolerance was selectively induced against those epitopes expressed in an immunogenic form by endogenously produced, pCSP-encoded antigen.

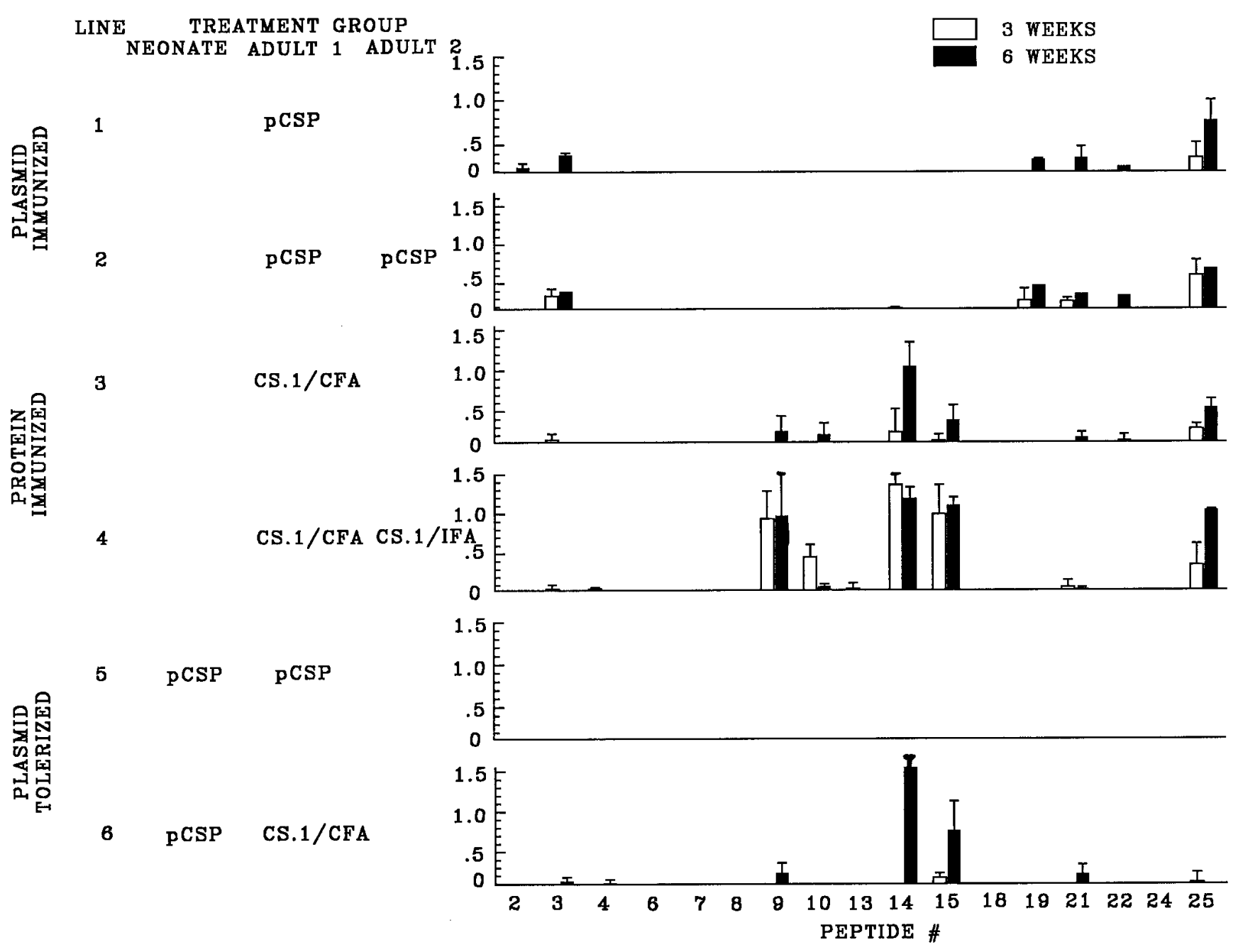

Figure 2. A series of synthetic peptides spanning the length of the P. yoelii CS protein (23) were used to analyze the epitope specificity of the antibody response elicited by pCSP or CS.1/CFA in BALB/c mice. Mice were treated as neonates at 2-5 d of age, primed at $7 \mathrm{wk}$ of age, and boosted at 3 mo of age. The same pattern of antibody responsiveness was observed in mice immunized and/or boosted up to 9 mo after neonatal administration of pCSP. Data represent the mean optical density \pm SD of sera from three to nine animals/group individually tested using a peptide-specific ELISA (see Fig. 1 for details). 
A. IN VIVO
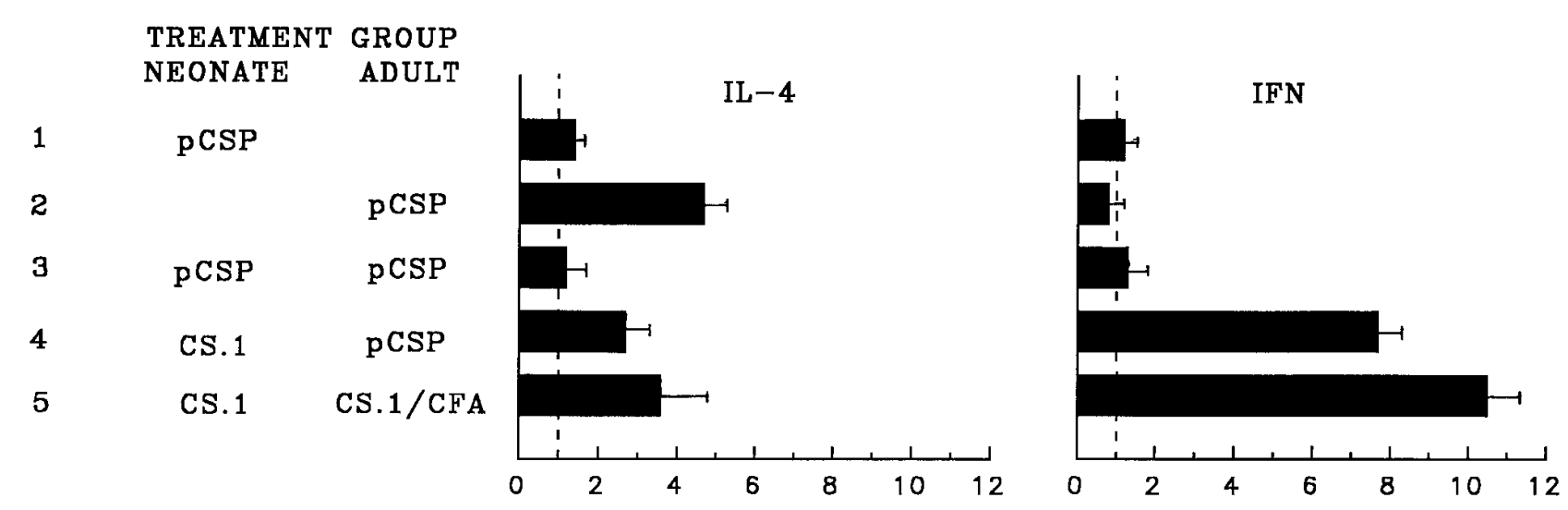

B. IN VITRO

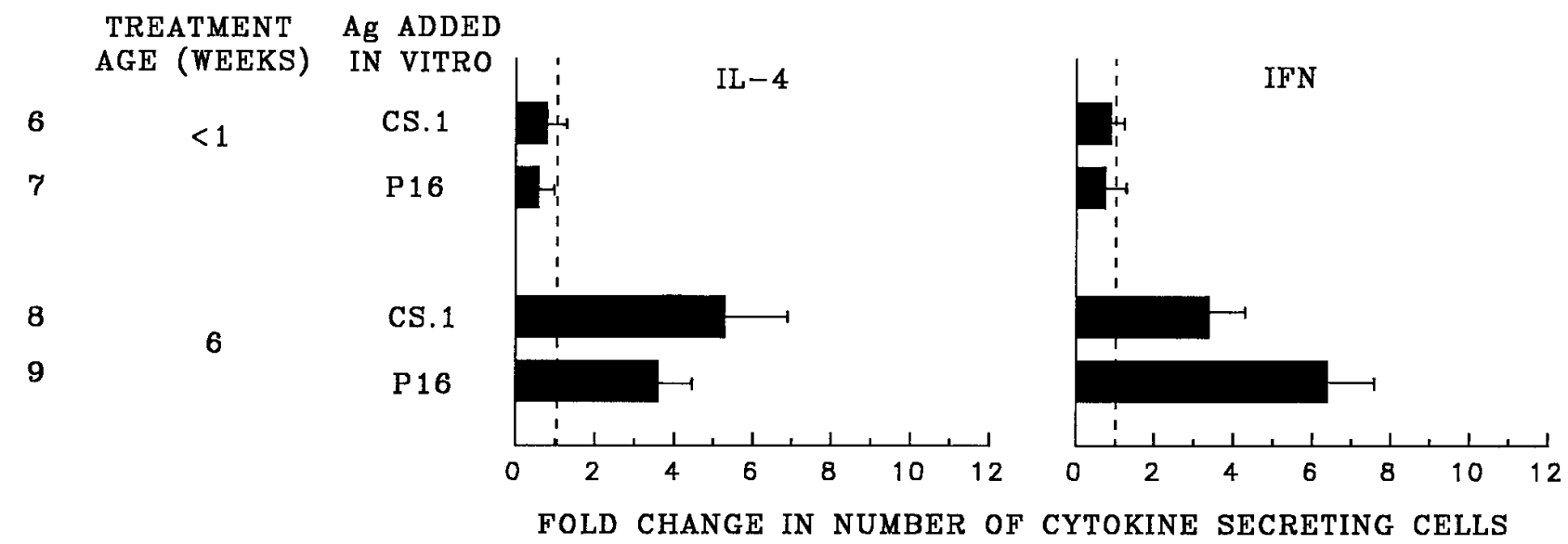

Figure 3. Cytokine ELIspot assays were used to detect individual IL-4 and IFN $\gamma$ secreting BALB/c spleen cells $(34,35)$. Panel $A$ examines the number of spleen cells actively secreting cytokine in vivo in mice $3 \mathrm{wk}$ after pCSP or CS.1 treatment when compared with age-matched controls (average $\pm \mathrm{SD}$ of three individually studied mice/group). There were $\sim 50 / 10^{6}$ spleen cells spontaneously secreting IL-4 and $120 / 10^{6}$ spontaneously secreting IFN $\gamma$ in control mice. Panel $B$ examines spleen cells from pCSP-treated mice ( $3 \mathrm{wk}$ after treatment) that could be stimulated to secrete cytokine after $10 \mathrm{~h}$ of in vitro stimulation with $10 \mu \mathrm{g} / \mathrm{ml}$ of CS.1 or P16 (a stimulatory T cell epitope on PyCSP, [23, 24]). Spleen cells from untreated mice acted as age-matched controls. Data represent the average \pm SD of six individually studied mice/group.

Effect of pCSP on cytokine production. The pattern of cytokines elicited by the administration of pCSP was then examined. Our lab previously showed that adult BALB/c mice immunized with pCSP significantly increased their production of the Th 2 cytokine IL-4 (Fig. 3, line 2 and [21]), while secondary immunization of adults induced increased secretion of the Th1 cytokine IFN $\gamma$ (see reference 21). Consistent with those results, mice treated as neonates with exogenous CS.1 protein and then immunized as adults with pCSP or CS.1/CFA produced both IL-4 and IFN $\gamma$ in vivo (Fig. 3, lines 4 and 5). In contrast, no activation of IL-4 or IFN $\gamma$-secreting cells was detected in mice tolerized to pCSP as neonates and then immunized with that DNA vaccine as adults (Fig. 3, lines 1 and $3)$. These findings suggest that classical neonatal tolerance (characterized by the absence of antibody or cytokine production) was elicited against epitopes present on the pCSP-encoded antigen.

An antigen restimulation assay was used to determine whether memory $\mathrm{T}$ cells were present in tolerized mice. Our lab previously showed that such cells are rapidly induced to release Th1 or Th 2 cytokines when exposed to their target antigen in vitro (24). Initial experiments examined the response of spleen cells from adult BALB/c mice immunized with pCSP. As seen in Fig. 3 (lines 8 and 9), exposure to intact CS.1 protein or an immunogenic 16-AA peptide from that protein stimulated the production of both IL- 4 and IFN $\gamma$. In contrast, splenocytes from neonatally tolerized mice did not respond to in vitro stimulation by full length CS.1 or the immunostimulatory Th peptide (Fig. 3, lines 6 and 7). These findings support the conclusion that pCSP is a toleragen rather than an immunogen in neonatal mice.

Effect of the pCSP DNA vaccine on cytotoxic $T$ cell responses. A final series of experiments examined the capacity of neonatally tolerized mice to generate an antigen-specific CTL response. Sedegah et al. previously demonstrated that $\mathrm{T}$ cells from pCSP-immunized adult mice could lyse MHCmatched targets expressing the immunodominant CTL peptide present on the CSP protein (3). This CTL activity was de- 

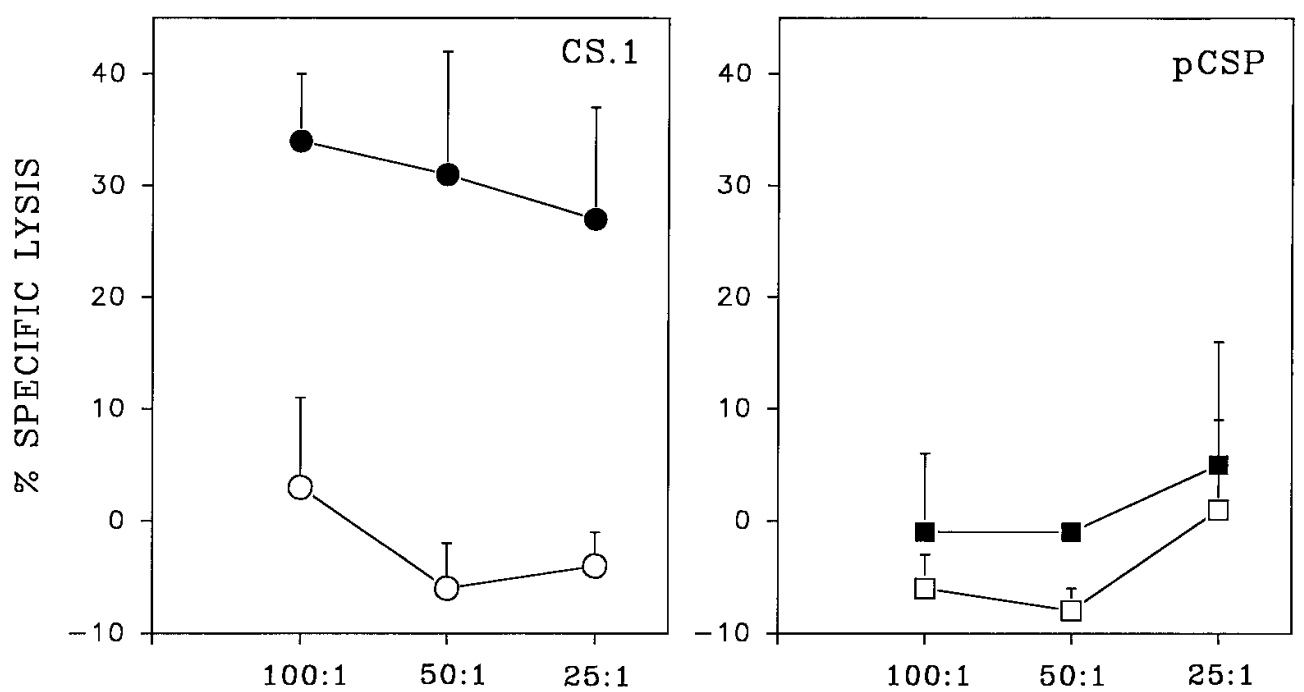

EFFECTOR:TARGET RATIO
Figure 4. 2-d-old BALB/c mice were treated with CS.1 or pCSP and immunized $6 \mathrm{wk}$ later with pCSP. At week nine, spleen cells were stimulated in vitro for $5 \mathrm{~d}$ with a synthetic CTL peptide from PyCSP and then assayed for cytolytic activity against MHC-matched P815 cells (solid symbols) or MHCincompatible EL4 cells (open symbols) pulsed with the same or an irrelevant peptide, as described $(3,23)$. Results represent the average \pm SD of three independently studied mice/ group. tected by restimulating antigen-reactive lymphocytes in vitro, and then monitoring their cytotoxicity against peptide-pulsed targets. We found that mice treated with CS.1 protein as neonates and then immunized as adults with pCSP mounted a strong cytotoxic response against peptide-pulsed MHC-matched targets (Fig. 4, left). Unpulsed targets, and peptide-pulsed targets expressing the wrong MHC type, were not lysed (Fig. 4 and data not shown). In contrast, spleen cells from mice tolerized as newborns and immunized as adults with pCSP were unable to generate CTL against MHC-matched peptide-pulsed targets, despite in vitro restimulation with an optimally immunogenic CTL peptide (Fig. 4, right).

\section{Discussion}

The development of vaccines capable of preventing bacterial, viral and parasitic infections is an important goal of biomedical research. DNA vaccines offer great promise towards this goal, since they are easy to construct and manufacture, and are cost competitive with conventional attenuated and subunit vaccines. Since the protein encoded by a DNA vaccine is produced endogenously and expressed in the context of self $\mathrm{MHC}$, this form of vaccination is particularly efficient at eliciting protective CTL as well as humoral immune responses in adult animals $(1,8)$. This report examines the effect of DNA vaccination of young mice, since many human vaccines are designed for use in children and newborns. Our results suggest that this mode of antigen presentation is capable of inducing neonatal tolerance rather than immunity.

Our research examined the pCSP DNA vaccine, which encodes the circumsporozoite protein of the $P$. yoelii malaria parasite. Previous studies showed that immunization of adult $\mathrm{BALB} / \mathrm{c}$ mice with this vaccine induced protective immunity against subsequent challenge with live sporozoites (3). Yet when this DNA vaccine was administered to 2-5 d-old mice, we found that long-lasting neonatal tolerance (persisting for $>9 \mathrm{mo}$, the duration of experimental follow-up) resulted. Mice tolerized as newborns were unable to mount antibody, cytokine or cytotoxic $\mathrm{T}$ cell responses against the pCSP-encoded antigen when challenged with DNA vaccine as adults. The induction of tolerance was critically dependent on both the age at which the vaccine was administered and the presence of the PyCSP-encoding gene. The pCSP vaccine did not induce tolerance when administered to mice $>2$ wk of age. In addition, tolerance was never elicited by exogenous CS.1 protein or by 1012 plasmid lacking the PyCSP-encoding insert. This set of findings suggests that tolerance is selectively elicited when the neonatal immune system is confronted with an endogenously produced DNA vaccine-encoded antigen. The mechanism underlying this tolerance induction is uncertain, and remains a topic of ongoing research. Variables including the site, quantity, duration, and/or MHC processing/presentation of relevant antigenic determinants may be involved.

Several recent reports challenge the existence of neonatal tolerance, claiming instead that newborns actively mount an immune response against foreign antigens but that their response differs in cytokine and/or antibody profile from that elicited in adults $(25,26)$. Our results do not support such a hypothesis, since pCSP-tolerized mice were unable to mount either Th1 (IFN $\gamma$ ) or Th2 (IL-4) cytokine responses when challenged with pCSP in vitro or in vivo. In contrast, adult mice immunized and/or boosted with this DNA vaccine were stimulated to secrete both types of cytokine. The profound state of neonatal tolerance observed following pCSP vaccination resembles classical self tolerance (27-30). We hypothesize that the neonatal responses reported by other authors (whose studies did not involve DNA vaccines) may represent incomplete tolerance induction.

It has also been reported that tolerance susceptibility is primarily determined by the maturity of the host's antigen presenting cells (31). Since neonatal tolerance was selectively induced by the pCSP DNA vaccine rather than by exogenous CS.1 protein, we conclude that the concentration and/or mode of antigen presentation, rather than the maturity of resident antigen presenting cells, plays the more important role in tolerance susceptibility. Of note, we were unable to elicit neonatal tolerance against the CS.1 protein injected i.m. or i.p., even at doses up to $100 \mu \mathrm{g} / \mathrm{animal}$ (data not shown). Other factors 
(such as the route of immunization, nature, or concentration of the encoded antigen, and the presence of immunostimulatory determinants in the plasmid backbone) may also affect the neonate's response, since not all DNA vaccines are toleragenic $(32,33)$.

Animal studies and preliminary results from phase I trials indicate that DNA vaccines can be safely administered to human adults. Yet our findings demonstrate that DNA vaccines have the potential to induce tolerance rather than immunity in newborns. Ongoing studies will determine whether pCSP-tolerized neonates are at increased risk of infection by live sporozoites. Given the importance of vaccines in preventing childhood diseases, DNA vaccines targeted for use in children or newborns should be thoroughly tested for safety and efficacy in young animals, whose immune system reflects the functional activity and maturational age of the targeted human population.

\section{Acknowledgments}

The assertions herein are the private ones of the authors and are not to be construed as official or as reflecting the views of the Food and Drug Administration or the U.S. Navy or the Naval service at large. The experiments reported herein were conducted according to the principles set forth in the Guide for the Care and Use of Laboratory Animals. 1985. Institute of Laboratory Animal Resources, National Research Council, DHHS, Publ. (National Institutes of Health) 86-23.

This work was supported by a grant from the National Vaccine Program and the Naval Medical Research and Development Command work units 61102A 3M161102.BS13 AK111 and 62787A 3M162787.A870 AN121.

\section{References}

1. Ulmer, J.B., J.J. Donnelly, S.E. Parker, G.H. Rhodes, P.L. Felgner, V.J. Dwarki, S.H. Gromkoski, R.R. Deck, C.M. DeWitt, and A. Friedman. 1993. Heterologous protection against influenza by injection of DNA encoding a viral protein [see comments]. Science (Wash. DC). 259:1745-1749.

2. Cox, G.J., T.J. Zamb, and L.A. Babiuk. 1993. Bovine herpesvirus 1: immune responses in mice and cattle injected with plasmid DNA. J. Virol. 67: $5664-5667$.

3. Sedegah, M., R. Hedstrom, P. Hobart, and S.L. Hoffman. 1994. Protection against malaria by immunization with plasmid DNA encoding circumsporozoite protein. Proc. Natl. Acad. Sci. USA. 91:9866-9870.

4. Wang, B., K.E. Ugen, V. Srikantan, M.G. Agadjanyan, K. Dang, Y. Refaeli, A. Sato, J. Boyer, W.V. Williams, and D.B. Weiner. 1993. Gene inoculation generates immune responses against human immunodeficiency virus type 1. Proc. Natl. Acad. Sci. USA. 90:4156-4160.

5. Tascon, R.E., M.J. Colston, S. Ragno, E. Stavropoulos, D. Gregory, and D.B. Lowrie. 1996. Vaccination against tuberculosis by DNA injection. Nat. Med. 2:888-892.

6. Wolff, J.A., R.W. Malone, P. Williams, W. Chong, G. Ascadi, A. Jani, and P.L. Felgner. 1990. Direct gene transfer into mouse muscle in vivo. Science (Wash. DC). 247:1465-1468.

7. Manthorpe, M., J.F. Cornefert, J. Hartikka, J. Felgner, A. Rundell, M. Margalith, and V. Dwarki. 1993. Gene therapy by intramuscular injection of plasmid DNA: studies on firefly luciferase gene expression in mice. Hum. Gene Ther. 4:419-431.

8. Robinson, H.L., D.M. Feltquate, M.J. Morin, J.R. Haynes, and R.G. Webster. 1995. DNA vaccines: a new approach to immunization. Int. J. Immunopharmacology. 17:79-83.

9. Tang, D., M. DeVit, and S.A. Johnston. 1992. Genetic immunization is a simple method for eliciting an immune response. Nature (Lond.). 356:152-154.

10. Silverstein, A.M., and S. Segal. 1975. The ontogeny of antigen-specific T cells. J. Exp. Med. 142:802-804.

11. Marodon, G., and B. Rocha. 1995. Activation and 'deletion' of self-reactive mature and immature $\mathrm{T}$ cells during ontogen of Mls-1a: implications for neonatal tolerance induction. Int Immunol. 6:1899-1904.

12. Silverstein, A.M. 1977. Ontogeny of the immune response: a perspective. In Development of Host Defenses. M.D. Cooper, editor. Raven Press, New York. 1-10.

13. Sarzotti, M., D.S. Robbins, and P.M. Hoffman. 1996. Induction of protective CTL responses in newborn mice by a murine retrovirus. Science (Wash. DC). 271:1726-1728.

14. Sterzl, J., and A.M. Silverstein. 1967. Developmental aspects of immunity. Adv. Immunol. 6:337-459.

15. Klinman, N.R., N.H. Sigal, E.S. Metcalf, S.K. Pierce, and P.J. Gearhart. 1976. The interplay of evolution and environment in B-Cell diversification. Cold Spring Harbor Symp. Quant. Biol. 41:165-173.

16. Klinman, N.R. 1972. The mechanism of antigenic stimulation of primary and secondary clonal precursor cells. J. Exp. Med. 136:241-260.

17. Klinman, D.M., Y. Ishigatsubo, and A.D. Steinberg. 1988. Acquisition and maturation of expressed $\mathrm{B}$ cell repertoires in normal and autoimmune mice. J. Immunol. 141:801-806.

18. Charoenvit, Y., S. Mellouk, C. Cole, R. Bechara, M.F. Leef, S.M. Dedegah, L. Yuan, F. Robey, R. Beaudoin, and S. Hoffman. 1991. Monoclonal, but not polyclonal, antibodies protect against Plasmodium yoelii sporozoites. J. Immunol. 146:1020-1025.

19. Klinman, D.M., and T.B. Nutman. 1994. ELIspot assay to detect cytokine-secreting murine and human cells. In Current Protocols in Immunology. J. E. Coligan, A. M. Kruisbeek, D. H. Margulies, E. M. Shevach, and W. Strober, editors. Greene Publishing Associates, Brooklyn, NY. 6.19.1-6.19.7.

20. Klinman, D.M., A. Yi, S.L. Beaucage, J. Conover, and A.M. Krieg. 1996. CpG motifs expressed by bacterial DNA rapidly induce lymphocytes to secrete IL-6, IL-12 and IFNg. Proc. Natl. Acad. Sci. USA. 93:2879-2883.

21. Mor, G., D.M. Klinman, S. Shapiro, E. Hagiwara, M. Sedegah, J.A. Norman, S.L. Hoffman, and A.D. Steinberg. 1995. Complexity of the cytokine and antibody response elicited by immunizing mice with Plasmodium yoelii circumsporozoite protein plasmid DNA. J. Immunol. 155:2039-2046.

22. Sedegah, M., C.H. Chiang, W.R. Weiss, S. Mellouk, M.D. Cochran, R.A. Houghtom, R.S. Beaudoin, D. Smith, and S.L. Hoffman. 1992. Recombinant pseudorabies virus carrying a plasmodium gene: herpesvirus as a new live viral vector for inducting T- and B-cell immunity. Vaccine. 10:578-584.

23. Weiss, W.R., S. Mellouk, R.A. Houghten, M. Sedegah, S. Kumar, M.F. Good, J.A. Berzofsky, L.H. Miller, and S.L. Hoffman. 1990. Cytotoxic T cell recognize a peptide from the circumsporozoite protein on malaria-infected hepatocytes. J. Exp. Med. 171:763-773.

24. Aoki, I., O. Aoki, and D.M. Klinman. 1995. Activation of IL-4 and IL-6 secreting cells by antigen. Cytokine. 8:806-814.

25. Fursthuber, T., H.C. Yip, and P.V. Lehman. 1996. Induction of Thi and Th2 immunity in neonatal mice. Science (Wash. DC). 271:1728-1730.

26. Singh, R.R., B.H. Hahn, and E.E. Sercarz. 1996. Neonatal peptide exposure can prime $\mathrm{T}$ cells and, upon subsequent immunization, induce their immune deviation: implications for antibody vs. T cell-mediated autoimmunity. $J$. Exp. Med. 183:1613-1622.

27. Billingham, R.E., L. Brent, and P.B. Medawar. 1953. Actively acquired tolerance of foreign cells. Nature (Lond). 172:603-605.

28. Spear, P.G., and G.M. Edelman. 1974. Maturation of the humoral immune response in mice. J. Exp. Med. 139:249-263.

29. Billingham, R.E., and W.K. Silvers. 1960. Studies on tolerance of the Y chromosome antigen in mice. J. Immunol. 85:14-26.

30. Roser, B.J. 1989. Cellular mechanisms in neonatal and adult tolerance. Immunol. Rev. 107:179-202.

31. Ridge, J.P., E.J. Fuchs, and P. Matzinger. 1996. Neonatal tolerance revisited: turning on newborn T cells with dendritic cells. Science (Wash. DC). 271:1723-1726.

32. Sarzotti, M., T.A. Dean, M.P. Remington, C.D. Ly, P.A. Furth, and D.S. Robbins. 1996. Induction of CTl responses in newborn mice by DNA immunization. Nucleic Acid Vaccines. (Abstr.).

33. Prince, A.M., R. Whalen, and B. Brotman. 1996. Successful DNA-based immunization of newborn chimpanzees. Nucleic Acid Vaccines. (Abstr.).

34. Shirai, A., K. Holmes, and D.M. Klinman. 1993. Detection and quantitation of cells secreting IL-6 under physiological conditions in BALB/c mice. $J$. Immunol. 150:793-799.

35. Shirai, A., V. Sierra, C.I. Kelly, and D.M. Klinman. 1994. Individual cells simultaneously produce both IL-4 and IL-6 under physiologic conditions in vivo. Cytokine. 6:329-336 